\title{
Activation of the mitogen-activated protein kinase (MAPK) signalling pathway in the liver of mice is related to plasma glucose levels after acute exercise
}

\author{
M. Hoene $\cdot$ H. Franken $\cdot$ L. Fritsche $\cdot$ R. Lehmann • \\ A. K. Pohl • H. U. Häring • A. Zell • E. D. Schleicher • \\ C. Weigert
}

Received: 24 September 2009 /Accepted: 17 December 2009/Published online: 2 March 2010

(C) Springer-Verlag 2010

\begin{abstract}
Aims/hypothesis We aimed to identify, in the liver of mice, signal transduction pathways that show a pronounced regulation by acute exercise. We also aimed to elucidate the role of metabolic stress in this response.

Methods C57Bl6 mice performed a $60 \mathrm{~min}$ run on a treadmill under non-exhaustive conditions. Hepatic RNA and protein lysates were prepared immediately after running and used for whole-genome-expression analysis, quantitative real-time PCR and immunoblotting. A subset of mice recovered for $3 \mathrm{~h}$ after the treadmill run. A further group of mice performed the treadmill run after having received a vitamin $\mathrm{C}$ - and vitamin E-enriched diet over 4 weeks.

Results The highest number of genes differentially regulated by exercise in the liver was found in the mitogenactivated protein kinase (MAPK) signalling pathway, with a pronounced and transient upregulation of the transcription
\end{abstract}

Electronic supplementary material The online version of this article (doi:10.1007/s00125-010-1666-3) contains supplementary material, which is available to authorised users.

M. Hoene $\cdot$ L. Fritsche $\cdot$ R. Lehmann • A. K. Pohl $•$ H. U. Häring $•$

E. D. Schleicher $\cdot$ C. Weigert $(\bowtie)$

Division of Endocrinology, Diabetology, Angiology, Nephrology, Pathobiochemistry and Clinical Chemistry, Department of Internal

Medicine, University Hospital of Tuebingen,

Otfried-Mueller-Straße 10,

D-72076 Tuebingen, Germany

e-mail: Cora.Weigert@med.uni-tuebingen.de

H. Franken · A. Zell

Wilhelm-Schickard-Institute for Computer Science,

Centre for Bioinformatics, University of Tuebingen,

Tuebingen, Germany factors encoded by c-Fos (also known as Fos), c-Jun (also known as Jun), FosB (also known as Fosb) and JunB (also known as Junb) and phosphorylation of hepatic MAPK. Acute exercise also activated the p53 signalling pathway. A major role for oxidative stress is unlikely since the antioxidant-enriched diet did not prevent the activation of the MAPK pathway. In contrast, lower plasma glucose levels after running were related to enhanced levels of MAPK signalling proteins, similar to the upregulation of Igfbpl and Pgc-1 $\alpha$ (also known as Ppargcla). In the working muscle the activation of the MAPK pathway was weak and not related to plasma glucose concentrations.

Conclusions/interpretation Metabolic stress evidenced as low plasma glucose levels appears to be an important determinant for the activation of the MAPK signalling pathway and the transcriptional response of the liver to acute exercise.

Keywords Carbohydrate metabolism - Exercise - Mouse

\begin{tabular}{|c|c|}
\hline \multicolumn{2}{|c|}{ Abbreviations } \\
\hline $\mathrm{Akt} / \mathrm{PKB}$ & $\begin{array}{l}\text { Serine/threonine protein kinase/protein } \\
\text { kinase B }\end{array}$ \\
\hline c-FOS & FBJ osteosarcoma oncogene \\
\hline c-JUN & Jun oncogene \\
\hline DUSP & Dual-specificity phosphatase \\
\hline ERK & Extracellular signal regulated kinase \\
\hline IGFBP1 & Insulin-like growth factor binding protein 1 \\
\hline JNK1 & Janus kinase 1 \\
\hline MAPK & Mitogen-activated protein kinase \\
\hline MDM2 & Murine double minute 2 oncogene \\
\hline PGC- $1 \alpha$ & $\begin{array}{l}\text { Peroxisome proliferator-activated receptor- } \gamma \\
\text { coactivator- } 1 \alpha\end{array}$ \\
\hline PPAR & Peroxisome proliferator-activated receptor \\
\hline
\end{tabular}




\section{Introduction}

Regular physical activity is known to have multiple health benefits, including maintenance of insulin sensitivity and cardiorespiratory fitness. The working skeletal muscle is clearly the organ most directly affected during physical activity. Therefore, researchers have focused mainly on the molecular response of the contracting muscle to exercise to elucidate the pathways responsible. Primary events induced in the muscle are the $\mathrm{Ca}^{2+}$ release from the sarcoplasmic reticulum, ATP consumption and the generation of reactive oxygen species, which lead to activation of signal transduction pathways. The activation of key kinases and mediators within these pathways-importantly, but not exclusively, AMP-activated protein kinase $[1,2], \mathrm{Ca}^{2+} /$ calmodulin-dependent kinases [3], atypical protein kinase C $[4,5]$, and mitogen-activated protein kinase (MAPK) [6] initiates the upregulation of glucose uptake, which is the acute metabolic effect, and also regulates the adaptive response of the muscle to exercise training. Several of these pathways are involved in the exercise-dependent activation of the transcriptional co-activator peroxisome proliferatoractivated receptor- $\gamma$ coactivator- $1 \alpha$ (PGC- $1 \alpha)[7,8]$ and induction of transcription factors nuclear factor- $\mathrm{K}$ light chain gene enhancer in B cells [9], FBJ osteosarcoma oncogene (c-FOS) and the jun oncogene (c-JUN) [10] in the working muscle. The exercise-dependent differential gene expression in the muscle is an important mechanism in the adaptation to regular physical activity. The transcriptional response of the muscle to one single bout of exercise or contraction as well as to exercise training has been studied extensively. The majority of the differentially expressed genes are involved in glucose and fatty acid metabolism [11-13], and in myogenesis [14], the regulation of transcription $[15,16]$ and the stress response [17].

The metabolic adaptation to exercise is not restricted to the working muscle. It has been recognised for decades that the liver, as a major regulator of glucose and lipid homoeostasis, also responds to acute exercise, and increased activities of the gluconeogenic enzymes as well as decreased lipogenic enzyme activities have been found [18-20]. Compared with the numerous reports on the transcriptional regulation of genes in the working muscle, remarkably little data on exercise-induced gene expression in the liver are available. Some studies have investigated the effects of long-term exercise training on hepatic expression of metabolic enzymes in obese or diabetic rodents [21-23]. The expression of genes involved in the regulation of glucose and fatty acid metabolism was also found to be regulated by one single bout of exercise. These studies showed upregulation of adiponectin receptor 1 and forkhead box O1 production [24], downregulation of fatty acid synthase [25] and induction of gluconeogenic enzymes and PGC- $1 \alpha$ [26]. The data suggest that the liver is involved in the metabolic adaptation to regular physical activity and that exercise regulates hepatic gene expression. However, less is known about the signal transduction pathways activated by acute exercise in the liver.

To obtain a global impression of the transcriptional response of the liver to one single bout of exercise, we studied the differential gene expression in the liver of mice immediately after a $60 \mathrm{~min}$ run on a treadmill under nonexhaustive conditions with whole-genome-expression analysis. In the liver, 536 genes were found to be differentially expressed immediately after cessation of running. The highest number of differentially expressed genes is implicated in the MAPK signalling pathway. Further studies revealed that increased expression of the genes encoding, or activation of, signalling molecules of this pathway is blunted after $3 \mathrm{~h}$ of recovery, and that expression was correlated to the plasma glucose levels after exercise and was not prevented by an antioxidant-enriched diet.

\section{Methods}

Materials The mouse Accupacer treadmill with motorised grade adjust was from Hugo Sachs Elektronik (MarchHugstetten, Germany). The LightCycler system was from Roche (Mannheim, Germany). The oligonucleotides were from Qiagen (Hilden, Germany) or Invitrogen (Karlsruhe, Germany). Antibodies against phosphorylated extracellular signal regulated kinase (phospho-ERK), phospho-p38 MAPK, ERK1/2 (p44/p42), p38 MAPK, phosphorylated janus kinase 1 (JNK1), phospho-ser-473 serine/threonine protein kinase/protein kinase B (Akt/PKB) and phosphoser-166-transformed mouse 3T3 cell murine double minute 2 (MDM2) oncogene were from Cell Signalling (Frankfurt, Germany). Antibodies against p53, MDM2 and insulin-like growth factor binding protein 1 (IGFBP1) were from Santa Cruz Biotechnology (Santa Cruz, CA, USA). Antibodies against JNK and Akt/PKB were from BD Biosciences (San Diego, CA, USA). The protease inhibitor mixture was from Roche.

Animal care and exercise protocol All animal experiments were conducted in accordance with the National Institutes of Health guidelines of laboratory animal care and were approved by the local governmental commission for animal research (Regierungspraesidium). Male C57BL/6 mice were purchased from Charles River Wiga (Sulzfeld, Germany) and kept under an inverted light-dark cycle (dark period 9:30-21:30 hours, light period 21:30-9:30 hours). All experiments were performed between 10:00 and 14:00 hours. Mice were habituated to treadmill running for $10 \mathrm{~min}$ at $5 \mathrm{~m} / \mathrm{min}$ and $5^{\circ}$ inclination twice during 1 and 
2 weeks prior to the bout of endurance exercise, which took place at 12 weeks of age. In the experiments, mice ran $60 \mathrm{~min}$ at $14 \mathrm{~m} / \mathrm{min}$ and $14^{\circ}$ inclination after $5 \mathrm{~min}$ warmup $\left(5 \mathrm{~m} / \mathrm{min}\right.$ and $5^{\circ}$ inclination) and were either killed immediately after the run or placed back in their cages for $3 \mathrm{~h}$ recovery. Sedentary mice remained in their cages. Mice attempting to rest were encouraged to continue running by gently tapping on their back. Mice of all groups had no access to food for the last $60 \mathrm{~min}$ before they were killed, either because of running or because of food withdrawal. In a further experiment, mice were fed a diet supplemented with $100 \mathrm{mg} / \mathrm{kg}$ vitamin $\mathrm{C}$ and $2,000 \mathrm{U} / \mathrm{kg}$ vitamin $\mathrm{E}$ or a control diet $(0 \mathrm{mg} / \mathrm{kg}$ vitamin $\mathrm{C}, 149 \mathrm{U} / \mathrm{kg}$ vitamin $\mathrm{E})$ based on C1000, Altromin (Lage, Germany) for 4 weeks before the acute exercise experiment. For the experiments not involving dietary modifications, standard chow was purchased from ssniff Spezialdiäten (Soest, Germany). All animals were anaesthetised with an intraperitoneal injection of ketamine $(150 \mathrm{mg} / \mathrm{kg}$ body weight $)$ and xylazine $(10 \mathrm{mg} /$ $\mathrm{kg}$ body weight) and killed by decapitation. Tissues were immediately removed and frozen in liquid nitrogen or directly processed for protein analysis.

Blood variables NEFA and insulin were measured in the EDTA-plasma collected after decapitation. NEFA concentrations were detected by an enzymatic method (ADVIA 1650, Siemens Healthcare Diagnostics, Fernwald, Germany), and insulin levels by radio-immunoassay (Linco Research [now Millipore], St Charles, MO, USA). Glucose was quantified in capillary blood taken from the tail vein using an Accu-Chek Aviva glucometer (Roche).

RNA isolation and microarray analysis Frozen tissue was homogenised in a TissueLyser (Qiagen) and RNA was extracted with the RNeasy Fibrous Tissue Kit (Qiagen) according to the manufacturer's instructions. Total RNA from four mice per group was pooled, amplified with the MessageAmp Kit II (Ambion, Austin, TX, USA) and hybridised to a MouseRef-8 v1.0 Expression BeadChip array (Illumina, San Diego, CA, USA) by the customer service programme of the Microarray Facility, Tuebingen. The service also included image processing, quality control of the arrays, scaling and normalisation of signal values, comparison between experimental and baseline datasets and generation of lists with differentially regulated genes. Only expression changes $\leq 0.5$-fold and $\geq$ twofold were considered relevant.

We implemented automated access to the Kyoto Encyclopedia of Genes and Genomes (KEGG) database [27] to retrieve information about pathways and the participation of the inspected genes in pathways. As a measure of the impact of the single bout of exercise on a particular pathway we counted the number of differentially expressed genes in each pathway and summed the absolute fold change values of the differentially expressed genes participating in that pathway.

$R N A$ isolation, $R T-P C R$ and real-time quantitative $P C R$ analysis Reverse transcription of total RNA $(1 \mu \mathrm{g})$ was performed in a volume of $20 \mu \mathrm{l}$ using random hexamer primers with the First strand cDNA synthesis kit for RTPCR (Roche). Aliquots $(2 \mu \mathrm{l})$ were then submitted to online quantitative PCR with the LightCycler system with SYBR green using FastStart DNA-MasterSYBR Green I (Roche) or the QuantiFast SYBR Green PCR Kit (Qiagen). The primer sets used and cycling conditions are described in the Electronic supplementary material (ESM). The quantitative PCR was performed in a volume of $20 \mu \mathrm{l}: 2 \mu \mathrm{l}$ FastStart DNA-MasterSYBR Green I, $\mathrm{MgCl}_{2} 4 \mathrm{mmol} / \mathrm{l}$, and primers at a concentration of $1 \mu \mathrm{mol} / 1$ or according to the instructions of the QuantiTect Primer Assay and QuantiFast SYBR Green PCR Kit.

The mRNA content is given in arbitrary units. Normalisation to $\beta$-actin mRNA content gave results comparable with non-normalised values.

Tissue lysates and western blotting Liver and muscles were removed as described and immediately homogenised at $4^{\circ} \mathrm{C}$ in lysis buffer $(50 \mathrm{mmol} / \mathrm{l}$ Tris $\mathrm{pH} 7.6,150 \mathrm{mmol} / \mathrm{l} \mathrm{NaCl}$, $1 \%$ [vol./vol.] Triton-X100 containing protease and phosphatase inhibitors). After solubilisation on ice for $30 \mathrm{~min}$, the homogenates were clarified by three rounds of centrifugation $(10 \mathrm{~min}$ at $12,000 \mathrm{~g})$. SDS-PAGE, $7.5 \%$ (wt/vol.), and western blotting were performed as previously described [26].

Statistical analysis Means \pm SEM were calculated and groups of data were compared using the Student's $t$ test. Regression analysis was performed with the statistical software package JMP 4.0 (SAS Institute, Cary, NC, USA). The statistical significance was set at $p<0.05$.

\section{Results}

Microarray expression analysis In an initial attempt to screen for the genes and pathways most affected in the liver by one single bout of exercise, whole-genome-expression analysis was conducted on the liver RNA obtained from mice immediately after a single treadmill run for $60 \mathrm{~min}$ at high aerobic intensity. We found 352 genes upregulated more than twofold, and 184 genes downregulated more than twofold in the liver (giving a total of 536 genes affected), with 37 genes regulated more than tenfold. Analysis of the pathways potentially affected by the differential gene expression showed that-apart from 
metabolic pathways including fatty acid metabolism, glycolysis/gluconeogenesis, arachidonic acid metabolism, insulin signalling and peroxisome proliferator-activated receptor (PPAR) signalling - the MAPK signalling pathway comprised the highest number of differentially expressed genes and highest sum of absolute fold change values (Table 1). The complete list of regulated genes in the MAPK signalling pathway is shown in Table 2. In further studies we focused primarily on the activation of the MAPK pathway by acute exercise.

Acute exercise induces pronounced and transient expression of hepatic genes of the MAPK signalling pathway To verify the data of the microarray expression analysis and to study the expression of genes in the recovery phase, mice completed the $60 \mathrm{~min}$ run on the treadmill followed by a $3 \mathrm{~h}$ recovery phase. We studied the expression of c-Fos, c-Jun and Gadd45 (also known as Gadd45g) as well as the related transcription factors $\operatorname{JunB}$ (also known as Junb) and FosB (also known as Fosb). All five genes showed similar kinetics: an increased expression immediately after $60 \mathrm{~min}$ of exercise, which dropped to basal values after $3 \mathrm{~h}$ of recovery phase (Fig. 1a-e). Evaluation of the expression of these genes in oxidative

Table 1 Hepatic signalling pathways after acute exercise in descending order of the number of participating differentially expressed genes

\begin{tabular}{llc}
\hline Pathway description & $\begin{array}{l}\text { No. of } \\
\text { differentially } \\
\text { expressed genes }\end{array}$ & $\begin{array}{l}\text { Summed values } \\
\text { for absolute fold } \\
\text { change }\end{array}$ \\
\hline MAPK signalling pathway & 16 & 220.0 \\
$\begin{array}{l}\text { Cytokine-cytokine receptor } \\
\text { interaction }\end{array}$ & 8 & 41.2 \\
PPAR signalling pathway & 7 & 26.8 \\
Insulin signalling pathway & 7 & 28.7 \\
Focal adhesion & 6 & 16.5 \\
Arachidonic acid metabolism & 5 & 18.3 \\
Toll-like receptor signalling & 5 & 43.5 \\
pathway & 5 & 13.5 \\
Wnt signalling pathway & 5 & 18.2 \\
Adipocytokine signalling & 5 & 15.5 \\
pathway & 5 & 11.7 \\
VEGF signalling pathway & 5 & 19.4 \\
Jak-STAT signalling pathway & 4 & 10.3 \\
Calcium signalling pathway & 4 & 10.7 \\
GnRH signalling pathway & 4 & 12.1 \\
Glycolysis/gluconeogenesis & 4 & 17.7 \\
p53 signalling pathway & 4 & 15.0 \\
Axon guidance & 4 & \\
Fatty acid metabolism & 4 & \\
\hline
\end{tabular}

GnRH, gonadotrophin-releasing hormone; Jak-STAT, janus kinasessignal transducers and activators of transcription; VEGF, vascular endothelial growth factor
Table 2 Differentially expressed genes of the hepatic MAPK signalling pathway

\begin{tabular}{|c|c|c|}
\hline Gene & Description & Fold change \\
\hline Gadd $45 \gamma$ & $\begin{array}{l}\text { Growth arrest and } \\
\text { DNA-damage-inducible } 45 \gamma\end{array}$ & 3.7 \\
\hline Dusp8 & Dual specificity phosphatase 8 & 2.9 \\
\hline Rasa2 & RAS p 21 protein activator 2 & 2.4 \\
\hline Dusp6 & Dual specificity phosphatase 6 & 2.9 \\
\hline Fos & FBJ osteosarcoma oncogene & 29.3 \\
\hline Mapk8 & Mitogen-activated protein kinase 8 & 3.4 \\
\hline Jun & Jun oncogene & 2.4 \\
\hline $\operatorname{Ill} \beta$ & Interleukin $1 \beta$ & 6.0 \\
\hline Hspa2 & Heat shock protein 2 & 2.5 \\
\hline Dusp4 & Dual specificity phosphatase 4 & 147.0 \\
\hline Mapkapk3 & $\begin{array}{l}\text { Mitogen-activated protein } \\
\text { kinase-activated protein kinase } 3\end{array}$ & 5.8 \\
\hline Mapkapk2 & $\begin{array}{l}\text { Mitogen-activated protein } \\
\text { kinase-activated protein kinase } 2\end{array}$ & 2.0 \\
\hline Ppm1 $\beta$ & $\begin{array}{l}\text { Protein phosphatase } 1 \mathrm{~B}, \\
\text { magnesium dependent, } \beta \text { isoform }\end{array}$ & -2.1 \\
\hline Gadd $45 \alpha$ & $\begin{array}{l}\text { Growth arrest and } \\
\text { DNA-damage-inducible } 45 \alpha\end{array}$ & -2.9 \\
\hline Rac2 & RAS-related C3 botulinum substrate 2 & -2.4 \\
\hline Mapk11 & Mitogen activated protein kinase 11 & -2.3 \\
\hline
\end{tabular}

soleus muscle revealed a similar regulation of $c$-Fos expression (Fig. 1f), while no significant upregulation of c-Jun, Gadd45 $\gamma$, FosB and JunB was found (Fig. 1g-j).

Activation of MAPKs in the liver by acute exercise Next we evaluated the activation of the MAPKs ERK, p38 MAPK and JNK by studying their phosphorylation state in liver extracts from exercised and sedentary mice and comparing the results with the phosphorylation of the kinases in white gastrocnemius or extensor digitorum longus muscles. As the soleus muscle was used for the preparation of RNA, and is a small muscle, it was not possible to monitor protein phosphorylation or abundance in soleus. The phosphorylation of p42/44 ERK was not increased in the liver, but we detected a robust and transient phosphorylation of an ERK isoform (Fig. 2a,b). The phosphorylation of this ERK isoform was not detectable in muscle tissue, while the phosphorylation of p44 ERK and to a lesser extent of p42 (data not shown) was clearly enhanced in the recovery phase (Fig. 2c,d). The phosphorylation of p38 MAPK was not induced by the treadmill exercise in all tissues studied (compare Fig. 2e,f,g). Phosphorylation of JNK could not be detected in white gastrocnemius muscle (data not shown), in line with the results from concentric non-damaging exercise [28]. In the liver, the phosphorylation signal of JNK was increased immediately after the treadmill run (Fig. 2h). These data further indicate activation of the MAPK signalling pathway by acute exercise in the liver. 

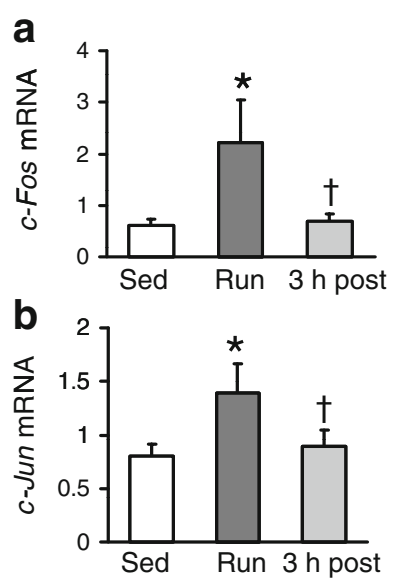

C
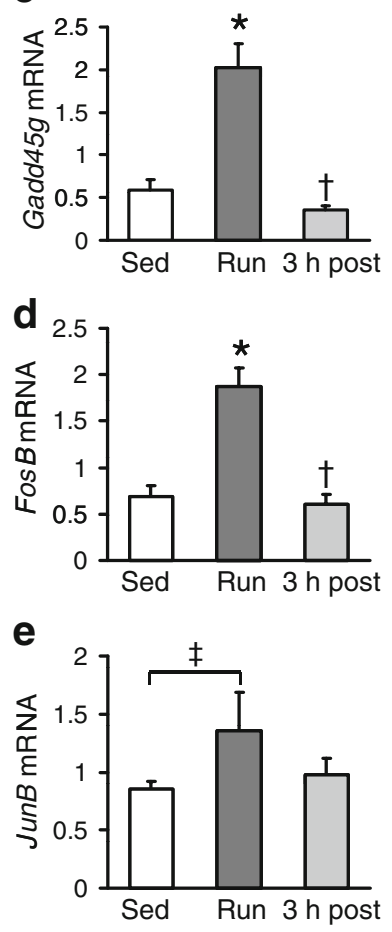

Fig. 1 mRNA expression of MAPK signalling proteins in liver and muscle. a $c-F o s$, b $c$-Jun, c Gadd $45 \gamma$, d FosB and e JunB mRNA content in liver tissue (a-e) or soleus muscle $(\mathbf{f}-\mathbf{j})$ of sedentary (Sed) and exercised (Run, $3 \mathrm{~h}$ post) mice. Values are shown as arbitrary units $\left(n=10\right.$, mean \pm SEM). ${ }^{*} p<0.05$ vs sedentary mice; ${ }^{\dagger} p<0.05$ vs run mice; ${ }^{\star} p=0.06$ vs sedentary mice

Microarray expression analysis indicated upregulation of dual-specificity phosphatase (DUSP) isoforms, which dephosphorylate MAPK. We studied the mRNA transcripts of Dusp1, which is expressed in high amounts in the liver, and Dusp6, the protein product of which shows high substrate specificity for ERK [29], and found a transient upregulation of mRNA levels of both phosphatase genes in the liver (Fig. 2i,j).

Activation of p53 and p53-target genes in the liver of the exercising mice The p53 signalling pathway was also found by our pathway analysis based on the microarray expression data (Table 1). We further verified the activation of this pathway by studying the expression of the gene encoding p53 inducible nuclear protein 1 (Trp53inp1), which was transiently upregulated in the liver (Fig. 3a). In line with expectations, we detected increased p53 protein levels in the liver of the exercised mice, particularly immediately after cessation of the exercise bout (Fig. 3b). Degradation of p53 is controlled by its interaction with MDM2, and phosphorylation of MDM2 at ser-166 activates MDM2 resulting in higher degradation of p53 [30]. This phosphorylation is controlled by the kinases ERK 1/2 and Akt/PKB [31]. We found significantly higher phosphorylation of MDM2 in the recovery phase, paralleled by an increased phosphorylation of ser-473 in Akt/PKB (Fig. 3c).

Effect of antioxidant-rich diet on the exercise-induced activation of hepatic MAPK signalling pathway We next aimed to identify the cellular stressors leading to the activation of the MAPK signalling pathway. Although we did not attempt to measure any biomarkers of oxidative stress, the increase in antioxidative gene expression found in the whole-genome-expression analysis (data not shown) suggests that our exercise protocol could have induced oxidative stress in the liver. Moreover, increased generation of reactive oxygen species in the liver of rats after acute exercise has been reported [32, 33]. Therefore, we evaluated the effect of an antioxidant-rich diet on the effects of the single bout of exercise. We fed the mice a diet enriched with vitamin $\mathrm{C}(100 \mathrm{mg} / \mathrm{kg}$ diet $)$ and vitamin $\mathrm{E}$ (2,000 U/kg diet) for 4 weeks, while the control group received an isoenergetic diet containing $149 \mathrm{U} / \mathrm{kg}$ vitamin $\mathrm{E}$. After 4 weeks, mice fed the vitamin-enriched diet had twofold higher vitamin E plasma values $(14.2 \pm 2.3 \mu \mathrm{mol} / \mathrm{l}$ vs $7.8 \pm 2.0 \mu \mathrm{mol} / \mathrm{l})$. Mice of both groups performed a single treadmill run for $60 \mathrm{~min}$ and gene expression was evaluated immediately after the exercise bout. The mRNA expression of the antioxidative enzymes superoxide dismutase 1 and 2 was not increased after the treadmill run while the mRNA expression of heme oxygenase 1 and metallothionein 1 was highly variable in both groups, with a higher mean value in the mice fed with the control diet (Fig. 4a-d). Sedentary mice of both diet groups showed no difference in $c$-Fos or Gadd45 $\gamma$ expression, and acute exercise induced expression of these genes to a similar extent independent of the diet (Fig. 4e,f). Moreover, the phosphorylation of the hepatic ERK isoform after exercise was not reduced (Fig. 4g). Thus, the antioxidative diet appeared not to prevent the exerciseinduced activation of the MAPK signalling pathway in the liver.

Involvement of glucose deprivation in the exercise-induced activation of hepatic MAPK signalling pathway The liver is 

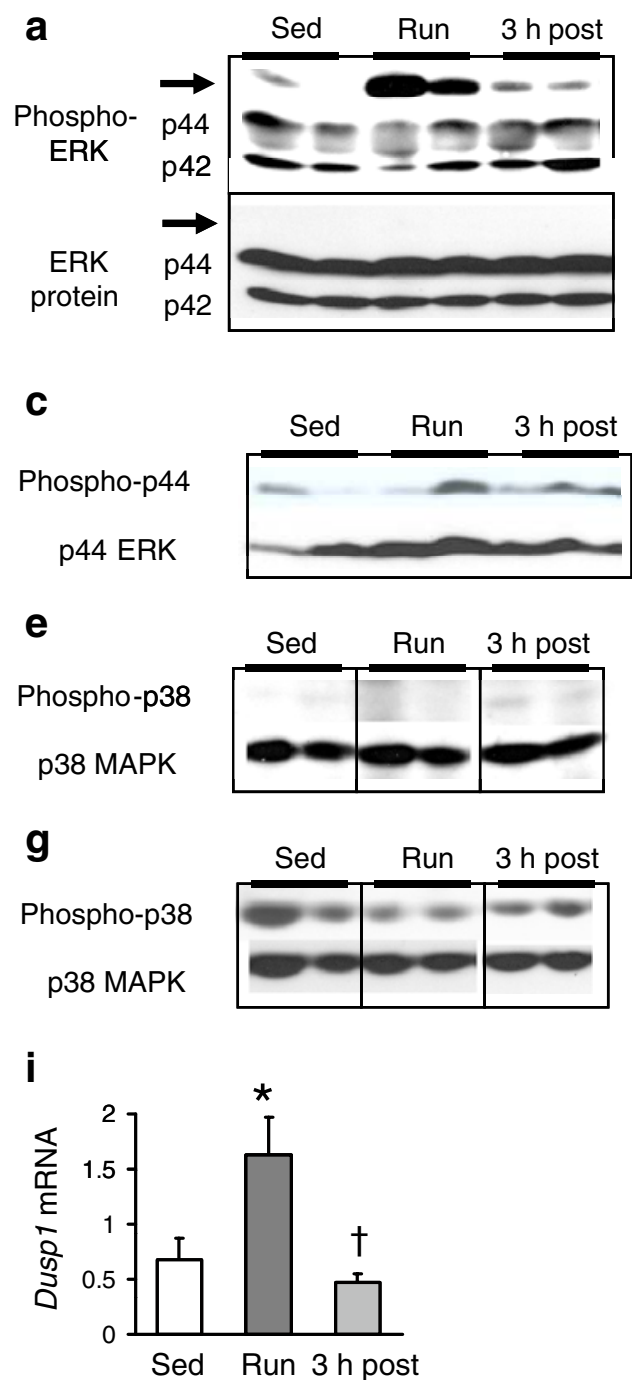

Fig. 2 Activation of MAPK in liver and muscle. a Detection of phosphorylated ERK and ERK protein of liver extracts of sedentary (Sed) and exercised (Run, $3 \mathrm{~h}$ post) mice and densitometric quantification shown as a histogram (b). The values are arbitrary units $\left(n=6\right.$, mean \pm SEM). ${ }^{*} p<0.05$ vs sedentary mice; ${ }^{\dagger} p<0.05$ vs run mice. The arrow indicates the position of phosphorylated ERK. Detection of phosphorylated ERK and ERK protein from $\mathbf{c}$ white gastrocnemius muscle and $\mathbf{d}$ extensor digitorum longus extracts. Detection of phosphorylated $\mathrm{p} 38$ and $\mathrm{p} 38$ protein from $\mathbf{e}$ liver, $\mathbf{f}$ white gastrocnemius muscle, and $\mathbf{g}$ extensor digitorum longus. $\mathbf{h}$ Detection

the major regulator of glucose homeostasis and responds to the increased glucose demand of the working muscle with glycogen breakdown, gluconeogenesis and glucose output into the circulation. We showed previously that the $60 \mathrm{~min}$ treadmill run of the mice resulted in glycogen depletion and induction of the gluconeogenic enzymes glucose-6phosphatase, phosphoenolpyruvate carboxykinase and PGC-1 $\alpha$ [26]. Despite these adaptations plasma glucose levels dropped significantly during the $60 \mathrm{~min}$ treadmill run (Fig. 5a). Plasma insulin levels were also reduced after running $(27.9 \pm 14.3$ vs $52.3 \pm 33.0 \mathrm{pmol} / 1$ in the sedentary

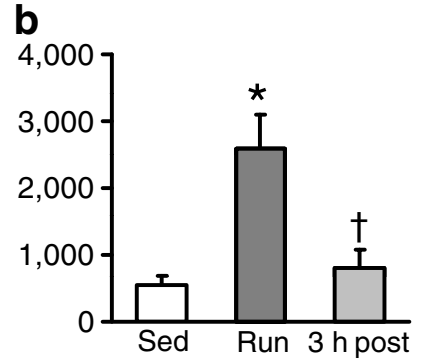

d
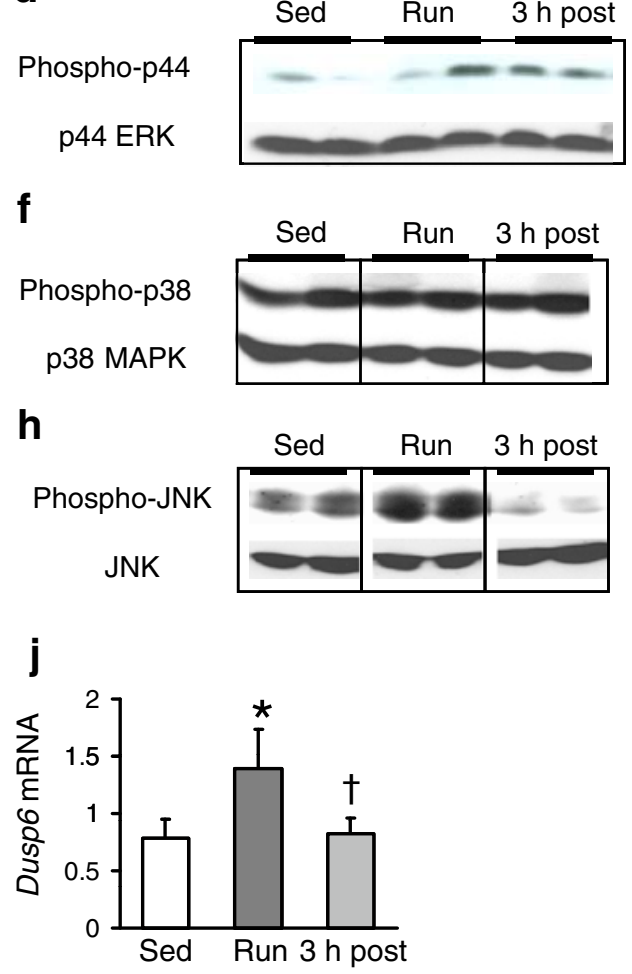

of phosphorylated JNK and JNK protein in liver. For (a)-(h), $150 \mu \mathrm{g}$ samples of protein from tissue lysates were separated by $10 \%$ SDSPAGE and immunoblotted with anti-phospho-ERK, ERK protein, anti-phospho-p38, p38 MAPK protein, anti-phospho-JNK and JNK protein antibodies. Shown are lysates from two individual animals from each group. Duspl (i) and Dusp6 (j) mRNA content in liver tissue from sedentary (Sed) and exercised (Run, $3 \mathrm{~h}$ post) mice. The values are shown as arbitrary units $(n=10$, mean \pm SEM $) .{ }^{*} p<0.05$ vs sedentary mice; ${ }^{\dagger} p<0.05$ vs run mice

group; $n=20 ; p=0.06)$. NEFA concentrations were increased ( $816 \pm 193$ vs $606 \pm 176 \mu \mathrm{mol} / 1 ; n=20 ; p=0.005)$. This metabolic challenge for the liver was reflected by the rapid and pronounced increase in Igfbpl mRNA (Fig. 5b) and IGFBP1 protein (Fig. 5c) levels. Moreover, high mRNA expression of $I g f b p l$ after exercise correlated with low plasma glucose levels (Fig. 5d) and low plasma insulin concentration after running $(p=0.006 ; R=0.61$; data not shown), but did not relate to the increase in NEFA after the run (data not shown). A similar relationship was found for low plasma glucose levels and expression of $P g c-1 \alpha$ 


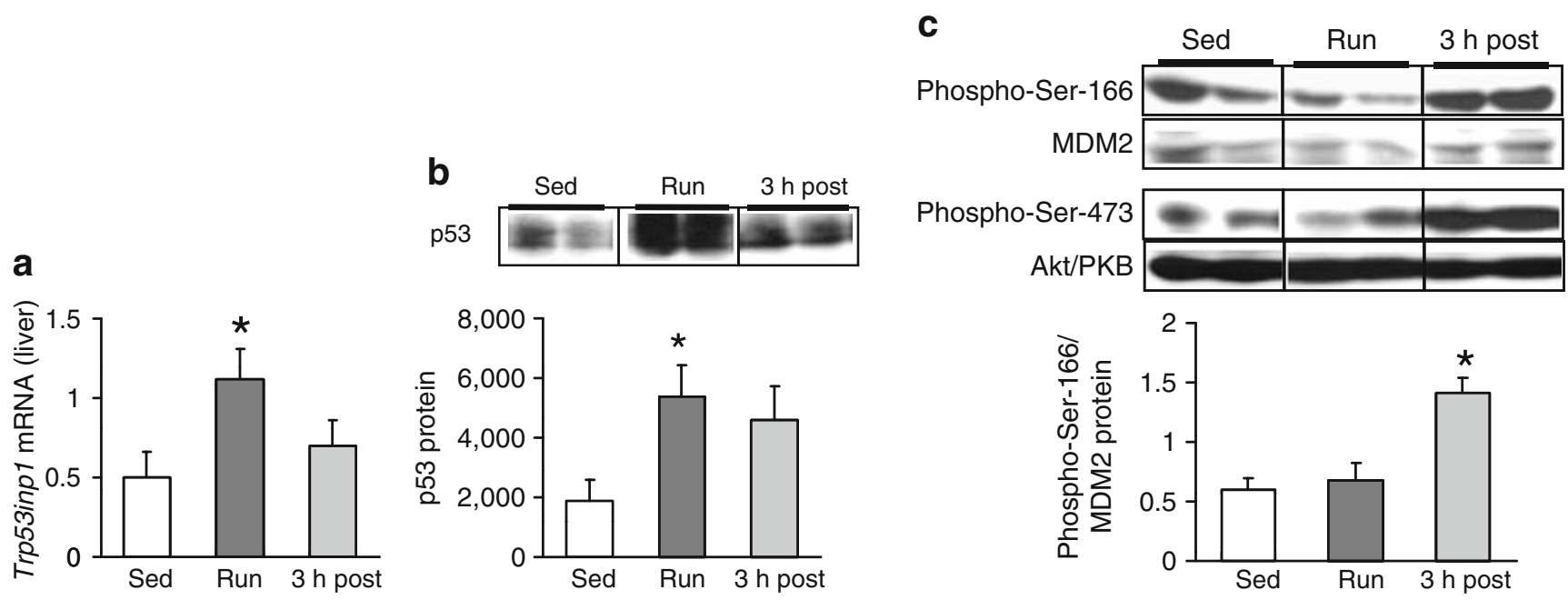

Fig. 3 Activation of p53 in the liver. a Trp53inp 1 mRNA content in liver tissue from sedentary (Sed) and exercised (Run, $3 \mathrm{~h}$ post) mice. Values are shown as arbitrary units $\left(n=10\right.$, mean \pm SEM). ${ }^{*} p<0.05$ vs sedentary mice. b Detection of p53 protein in liver extracts from sedentary (Sed) and exercised (Run, $3 \mathrm{~h}$ post) mice. $150 \mu \mathrm{g}$ protein samples from liver lysates were separated by $10 \%$ SDS-PAGE and immunoblotted with anti-p53 antibodies. Densitometric quantification of p53 is shown as a histogram. The values are arbitrary units $(n=6$,

(Fig. 5e), which strengthens the hypothesis that low plasma glucose concentrations are related to high expression of sensors of hepatic metabolic stress. Next we tested whether the concentrations of glucose, insulin or NEFA measured immediately after the $60 \mathrm{~min}$ treadmill run were related to the enhanced expression of the genes encoding the MAPK signalling molecules shown in Fig. 1. The mRNA expression of c-Fos, c-Jun, Gadd $45 \gamma$ and JunB correlated with low glucose concentrations after exercise (Fig. $5 \mathrm{f}-\mathrm{i}$ ), but not with glucose concentration before running, NEFA levels post exercise or, with the exception of Gadd45 $\gamma$ expression, with plasma insulin values post exercise (data not shown). No relationship of hepatic expression of Fos $B$ after running and glucose levels was found (data not shown). Of note, the increase in c-Fos expression in soleus muscle as shown in Fig. 1 did not correlate with the glucose concentration after the run (data not shown). These data suggest that the fall in plasma glucose concentration during exercise directly or indirectly induces not only the hepatic expression of metabolic genes, but also of genes encoding MAPK signalling molecules.

\section{Discussion}

The results obtained in this study clearly indicate that acute non-exhaustive exercise (e.g. $60 \mathrm{~min}$ of treadmill running) induces a pronounced and mainly transient mean \pm SEM). ${ }^{*} p<0.05$ vs sedentary mice. c Detection of phosphorylation of ser-166 of MDM2, of MDM2 protein, of phosphorylation of ser-473 of $\mathrm{Akt} / \mathrm{PKB}$, and of $\mathrm{Akt} / \mathrm{PKB}$ protein in liver lysates from sedentary (Sed) and exercised (Run, $3 \mathrm{~h}$ post) mice. Shown are lysates from two individual animals from each group. Densitometric quantification of MDM2 is shown as a histogram. The values are arbitrary units $\left(n=6\right.$, mean \pm SEM) ${ }^{*} p<0.05$ vs sedentary mice

activation of the hepatic MAPK signalling pathway in mice. First found in a whole-genome-expression analysis, the enhanced expression of the genes encoding MAPK signalling molecules c-Fos, c-Jun, Gadd $45 \gamma$ and related factors $\operatorname{Fos} B$ and $\operatorname{JunB}$ was verified by quantitative realtime PCR, and the activation of ERK and JNK was shown as enhanced phosphorylation of these proteins in hepatic tissues.

The phosphorylated form of ERK recognised by the antiphospho-Thr-202/Tyr-204 antibody is not identical to ERK1 or ERK2 and the molecular mass, which can be estimated from the immunoblots, is between 44 and $50 \mathrm{kDa}$. As the anti-p42/44 ERK antibody we used recognises a sequence in the C-terminal region of $\mathrm{p} 44$ ERK, the ERK isoform must differ in this region from $\mathrm{p} 42 /$ 44 ERK. Several isoforms of p42/44 ERK have been reported, such as the $46 \mathrm{kDa}$ ERK1b isoform with a 26 amino acid insertion between residues 340 and 341 of $\mathrm{p} 44$ ERK1 [34]. Although ERK1b would fulfil some criteria for being the phosphorylated ERK detected in the liver of mice after acute exercise, further studies are necessary to clarify the identity of this isoform. Of note, ERK1b could be regulated differently from p42/44 ERK. Particularly when p42/44 ERK is under tight phosphatase regulation it has been suggested that ERK1b could still be activated [34]. The upregulation of the MAPK phosphatases DUSP1 and DUSP6 immediately after exercise might provide an explanation for the lack of phosphorylation of ERK1/2 in the liver of the exercised mice. 

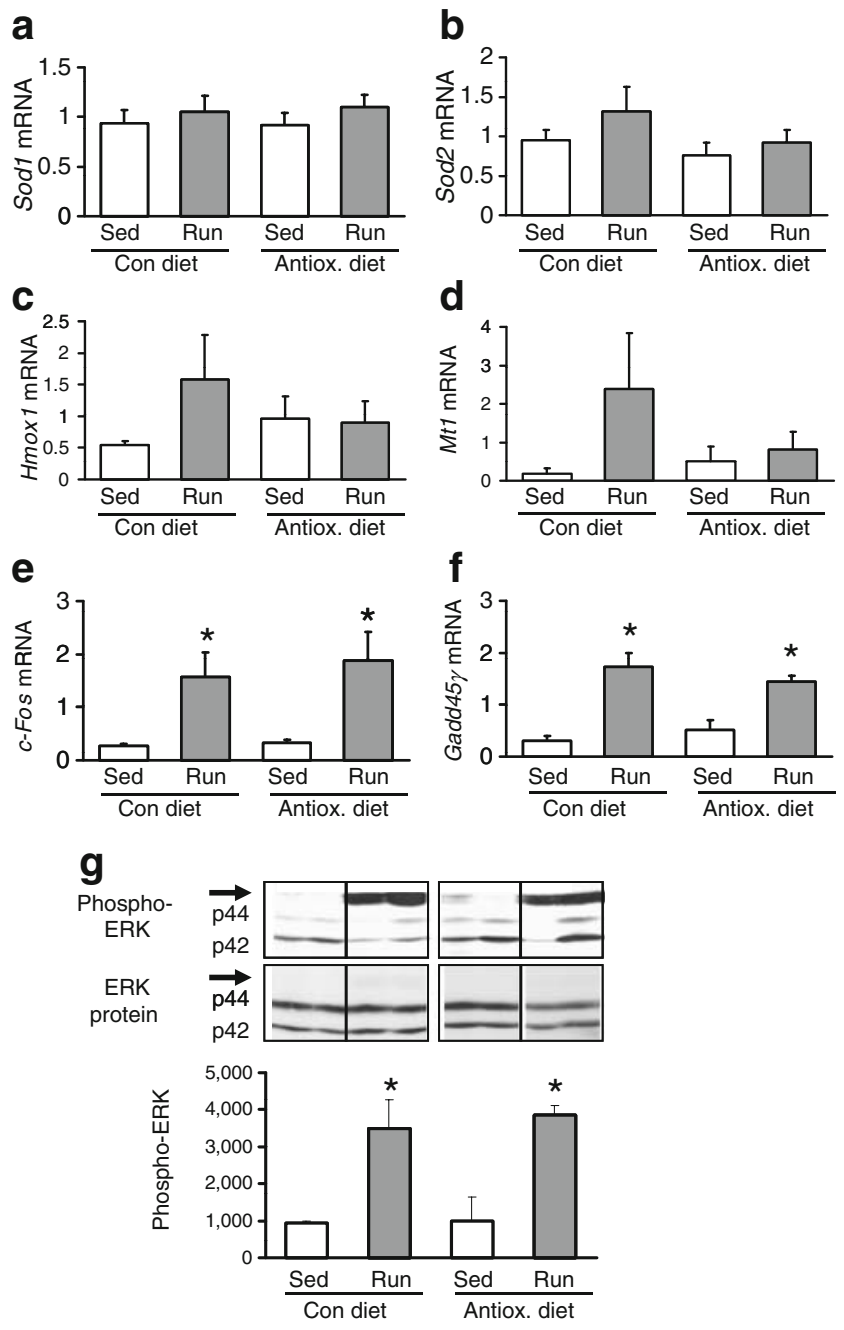

Fig. 4 Involvement of oxidative stress in the mRNA expression of genes encoding MAPK signalling proteins. a Sod1, b Sod2, c Hmoxl, d Mt1, e c-Fos, and f Gadd45 $\gamma$ mRNA content in the liver of sedentary (Sed) or exercised (Run) mice. One group of mice had received the control diet for 4 weeks (Con. diet), the second group had received antioxidant-enriched diet (Antiox. diet). g Detection of phosphorylated ERK and ERK protein in tissue extracts of sedentary (Sed) and exercised (Run) mice. $200 \mu \mathrm{g}$ samples of liver protein lysate were separated by $10 \%$ SDS-PAGE and immunoblotted with antiphospho-ERK and ERK protein antibodies. Shown are lysates from two individual animals from each group. The arrow indicates the position of phosphorylated ERK. Densitometric quantification is shown as a histogram. The values are arbitrary units $(n=5$, mean \pm SEM). ${ }^{*} p<0.05$ vs sedentary mice

An interesting question concerns the identification of the stimulus and the signalling pathway leading to exercisemediated induction of MAPK signalling proteins. We detected a clear relationship of the glucose concentrations after the exercise bout and the corresponding hepatic expression of c-Fos, c-Jun, Gadd45 $\gamma$ and JunB. A similar correlation was found for $I g f b p l$ and $P g c-1 \alpha$ expression. Prolonged exercise leads to a large increase in circulating IGFBP1 and the upregulation of IGFBP1 production in the liver in response to decreased glucose and insulin levels and glycogen depletion has been shown to be the main source of circulating IGFBP1 $[35,36]$. As we found no correlation of insulin with the expression of c-Fos, c-Jun, $J u n B$ and $P g c-1 \alpha$ it appears that in our model, glucose concentrations after running are a better predictor than insulin concentration. But which signal is elicited by the low plasma glucose concentration resulting in increased hepatic gene expression? Of note, this effect is specific for the liver, as no correlation was found between gene expression in the soleus muscle and plasma glucose.

The hormonal regulation of hepatic signalling pathways by the interplay of glucagon and insulin could be responsible for the regulation of hepatic gene expression. Unfortunately, we only measured glucagon plasma concentrations after running in a subset of eight mice, which is not sufficient to study any correlations. However, the lower glucose concentrations post-exercise might be a good correlate for glucagon action. Activation of hepatic glucagon receptors increases cAMP levels, leading to activation of protein kinase A, which activates ERK1/2 [37] and triggers several metabolic changes in the liver leading to enhanced hepatic glucose output [38]. Stimulation of alphaand beta-adrenoceptors by catecholamines also leads to activation of MAPK, and exercise is known to induce plasma norepinephrine and epinephrine concentrations [39]. However, strategies designed to specifically address the function of hepatic adrenergic stimulation during exercise, e.g. hepatic adrenoreceptor blockade in dogs [40], or inhibition of hepatic innervation in humans [41], do not support an important role in the stimulation of hepatic glucose output. Of course, this does not exclude the possibility that catecholamines are involved in the activation of hepatic MAPK signalling during exercise. Moreover, it is interesting to note that, independent of pancreatic hormones and catecholamine action, evidence exists that the fall in plasma glucose concentrations has stimulatory effects on the liver leading to enhanced glucose output [42, 43]. The results from these studies suggest that a signal related to the fall in plasma glucose, e.g. other metabolites released from the working muscle, or the decrease in glucose concentrations per se regulates the hepatic response to exercise.

In our study we also tested the hypothesis that exerciseinduced oxidative stress in the liver is implicated in the activation of the MAPK signalling pathway. Some studies reported the upregulation of variables of increased generation of reactive oxygen species such as lipid peroxidation, accumulation of nuclear 8-hydroxydeoxyguanosine, and induction of heat-shock proteins in the liver after acute exercise [32, 44]. Here, we studied the effect of a diet enriched with vitamin $\mathrm{C}$ and vitamin $\mathrm{E}$ for 4 weeks before the single exercise bout. Similar strategies have been shown 


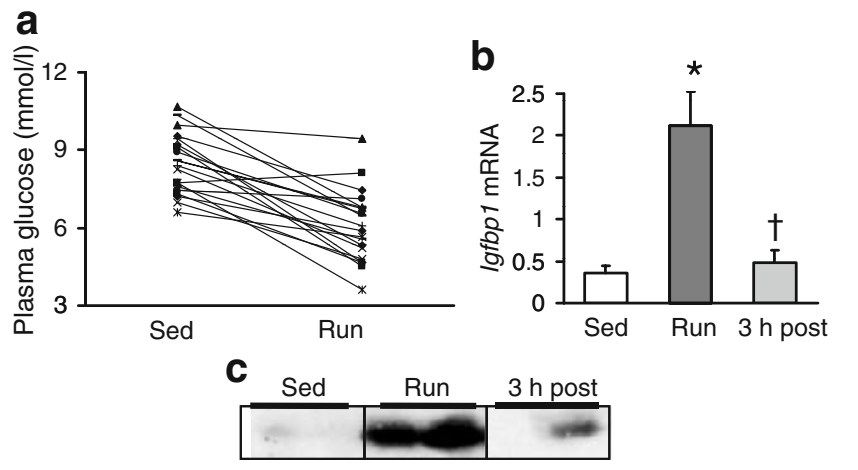

d

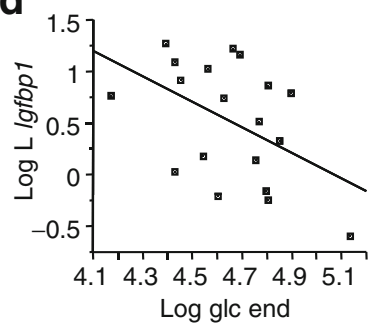

$\mathbf{f}$
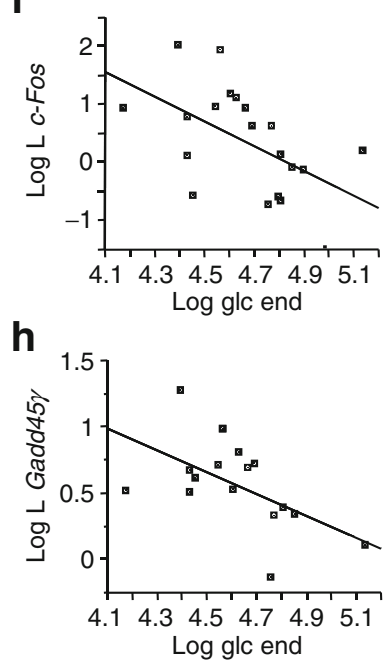

e
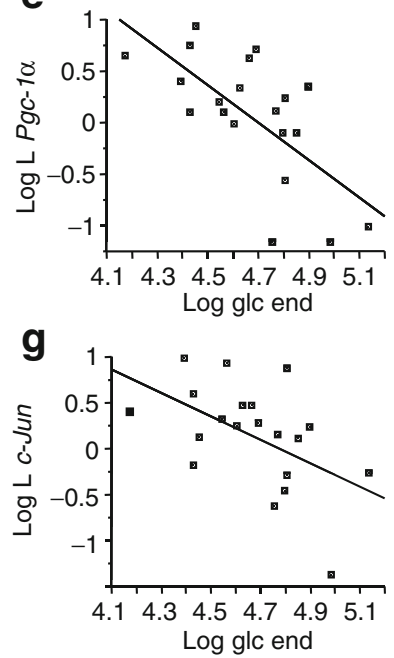

i

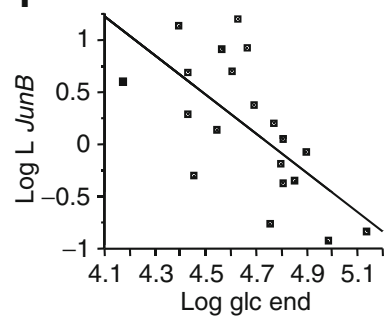

Fig. 5 Involvement of metabolic stress in the mRNA expression of MAPK signalling proteins. a Plasma glucose concentrations before and after running $(8.5 \pm 0.9 \mathrm{mmol} / 1$ vs $6.1 \pm 1.4 \mathrm{mmol} / 1[\mathrm{mean} \pm \mathrm{SE}] ; p<$ $0.001 ; n=20)$. b Igfbpl mRNA content in liver tissue of sedentary (Sed) and exercised (Run, $3 \mathrm{~h}$ post) mice. The values are shown as arbitrary units $\left(n=10\right.$, mean \pm SEM). ${ }^{*} p<0.05$ vs sedentary mice; ${ }^{\dagger} p<$ 0.05 vs run. c Detection of IGFBP1 protein in liver extracts from sedentary (Sed) and exercised (Run, $3 \mathrm{~h}$ post) mice. $150 \mu \mathrm{g}$ protein samples from liver lysates were separated by 10\% SDS-PAGE and immunoblotted with anti-IGFBP1 antibodies. Shown are lysates from two individual animals from each group. $\mathbf{d}-\mathbf{i}$ Relationship between

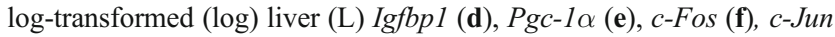
(g), Gadd45 $\gamma$ (h), and JunB (i) expression and plasma glucose (glc) concentrations immediately after exercise; $\mathbf{d} p=0.04, R=0.47(n=20)$; e $p=0.001, R=0.67(n=20) ; \mathbf{f} p=0.02, R=0.53(n=20) ; \mathbf{g} p=0.02, R=$ $0.51(n=20)$; h $p=0.03, R=0.56(n=16) ; \mathbf{i} p=0.002, R=0.66(n=20)$

to be successful in the prevention of exercise-induced oxidative stress in human muscle $[45,46]$ or in the reduction of oxidative stress in mice [47]. We did not detect differences in the expression of the genes coding for

MAPK signalling molecules c-Fos and Gadd45\%, which suggests that oxidative stress is not involved in the upregulation of these genes nor in the activation of MAPK. However, the induction of antioxidative proteins after the exercise bout was quite variable and we did not measure any biomarkers of oxidative stress. Therefore, we cannot exclude that the single treadmill run was not sufficient to induce oxidative stress in the liver nor could we verify the effectiveness of the antioxidant-rich diet.

After a $3 \mathrm{~h}$ recovery phase the expression values and phosphorylation status returned to values comparable with those in sedentary mice. Mice had access to food in the first $2 \mathrm{~h}$ of recovery phase, and plasma glucose levels increased from $6.1 \pm 1.4 \mathrm{mmol} / \mathrm{l}$ after the run to $7.4 \pm 1.0 \mathrm{mmol} / \mathrm{l}$ after the $3 \mathrm{~h}$ recovery phase, despite the withdrawal of food during the last hour. Thus, the mice could recover from the metabolic stress during running and the expression of MAPK signalling genes and metabolic regulators returned to basal values. Moreover, we found enhanced phosphorylation of Akt/PKB in the recovery phase, and this may be implicated in a negative feedback control to prevent further p53 protein activation via enhanced phosphorylation of ser166 in its negative regulator MDM2. Phosphorylation at this site is involved in activation of MDM2 and subsequent degradation of $\mathrm{p} 53[30,31]$.

Taken together, our data indicate that the liver of mice is strongly and rapidly affected by non-exhaustive treadmill exercise, resulting in a pronounced transcriptional response implicating particularly the MAPK signalling pathway. Activation of this pathway appears to be strongly influenced by a fall in plasma glucose levels, but not by oxidative stress. It will be of great interest to study whether the hepatic activation of the pathways found in the wholegenome-expression analysis is involved in the metabolic adaptation of the liver to exercise and beyond that in the beneficial effects of physical activity for the maintenance of insulin sensitivity. The activation of MAPK signalling in the skeletal muscle has been implicated in increased fatty acid uptake and oxidation [48]. Future studies will show how hepatic MAPK signalling is linked to the regulation of hepatic glucose and lipid metabolism during exercise or to the beneficial effects of regular physical activity on hepatic fat content as demonstrated in human studies [49, 50].

Acknowledgement This study was supported by grants from the Deutsche Forschungsgemeinschaft to C. Weigert (WE 4176/1) and to E. D. Schleicher (GRK 1302/1), and by the Kompetenznetz Diabetes mellitus (Competence Network for Diabetes mellitus) funded by the Federal Ministry of Education and Research to R. Lehmann, A. Zell and H. U. Häring (FKZ 01GI0803-04). We gratefully acknowledge the technical assistance of I. Riedlinger and E. Metzinger.

Duality of interest The authors declare that there is no duality of interest associated with this manuscript. 


\section{References}

1. Hardie DG (2004) AMP-activated protein kinase: a key system mediating metabolic responses to exercise. Med Sci Sports Exerc 36:28-34

2. Musi N, Yu H, Goodyear LJ (2003) AMP-activated protein kinase regulation and action in skeletal muscle during exercise. Biochem Soc Trans 31:191-195

3. Rose AJ, Kiens B, Richter EA (2006) $\mathrm{Ca}^{2+}$-calmodulin-dependent protein kinase expression and signalling in skeletal muscle during exercise. J Physiol 574:889-903

4. Perrini S, Henriksson J, Zierath JR, Widegren U (2004) Exerciseinduced protein kinase $\mathrm{C}$ isoform-specific activation in human skeletal muscle. Diabetes 53:21-24

5. Richter EA, Vistisen B, Maarbjerg SJ, Sajan M, Farese RV, Kiens B (2004) Differential effect of bicycling exercise intensity on activity and phosphorylation of atypical protein kinase $\mathrm{C}$ and extracellular signal-regulated protein kinase in skeletal muscle. J Physiol 560:909-918

6. Aronson D, Violan MA, Dufresne SD, Zangen D, Fielding RA, Goodyear LJ (1997) Exercise stimulates the mitogen-activated protein kinase pathway in human skeletal muscle. J Clin Invest 99:1251-1257

7. Lin J, Wu H, Tarr PT et al (2002) Transcriptional co-activator PGC-1 alpha drives the formation of slow-twitch muscle fibres. Nature 418:797-801

8. Pilegaard H, Saltin B, Neufer PD (2003) Exercise induces transient transcriptional activation of the PGC-1alpha gene in human skeletal muscle. J Physiol 546:851-858

9. Ji LL, Gomez-Cabrera MC, Steinhafel N, Vina J (2004) Acute exercise activates nuclear factor (NF)-kappaB signalling pathway in rat skeletal muscle. FASEB J 18:1499-1506

10. Puntschart A, Wey E, Jostarndt K et al (1998) Expression of fos and jun genes in human skeletal muscle after exercise. Am J Physiol 274:C129-C137

11. Jones TE, Baar K, Ojuka E, Chen M, Holloszy JO (2003) Exercise induces an increase in muscle UCP3 as a component of the increase in mitochondrial biogenesis. Am J Physiol Endocrinol Metab 284:E96-E101

12. Kraniou Y, Cameron-Smith D, Misso M, Collier G, Hargreaves M (2000) Effects of exercise on GLUT-4 and glycogenin gene expression in human skeletal muscle. J Appl Physiol 88:794-796

13. Neufer PD, Dohm GL (1993) Exercise induces a transient increase in transcription of the GLUT-4 gene in skeletal muscle. Am J Physiol 265:C1597-C1603

14. Yang Y, Creer A, Jemiolo B, Trappe S (2005) Time course of myogenic and metabolic gene expression in response to acute exercise in human skeletal muscle. J Appl Physiol 98:17451752

15. Russell AP, Hesselink MK, Lo SK, Schrauwen P (2005) Regulation of metabolic transcriptional co-activators and transcription factors with acute exercise. FASEB J 19:986-988

16. Baar K, Wende AR, Jones TE et al (2002) Adaptations of skeletal muscle to exercise: rapid increase in the transcriptional coactivator PGC-1. FASEB J 16:1879-1886

17. Essig DA, Borger DR, Jackson DA (1997) Induction of heme oxygenase-1 (HSP32) mRNA in skeletal muscle following contractions. Am J Physiol 272:C59-C67

18. Dohm GL, Kasperek GJ, Barakat HA (1985) Time course of changes in gluconeogenic enzyme activities during exercise and recovery. Am J Physiol 249:E6-E11

19. Barakat HA, Carpenter JW, Lennon YA, Hanna WR Jr, O'Brien KF, Dohm GL (1987) The effects of exercise on lipogenic enzyme activity and glyceride synthesis by liver homogenates of diabetic rats. Metabolism 36:983-987
20. Nizielski SE, Arizmendi C, Shteyngarts AR, Farrell CJ, Friedman JE (1996) Involvement of transcription factor C/EBP-beta in stimulation of PEPCK gene expression during exercise. Am J Physiol 270:R1005-R1012

21. Colombo M, Gregersen S, Kruhoeffer M et al (2005) Prevention of hyperglycemia in Zucker diabetic fatty rats by exercise training: effects on gene expression in insulin-sensitive tissues determined by high-density oligonucleotide microarray analysis. Metabolism 54:1571-1581

22. Lee KY, Kim SJ, Cha YS et al (2006) Effect of exercise on hepatic gene expression in an obese mouse model using cDNA microarrays. Obesity (Silver Spring) 14:1294-1302

23. Fiebig RG, Hollander JM, Ney D, Boileau R, Jeffery E, Ji LL (2002) Training down-regulates fatty acid synthase and body fat in obese Zucker rats. Med Sci Sports Exerc 34:1106-1114

24. Huang H, Iida KT, Sone H, Ajisaka R (2007) The regulation of adiponectin receptors expression by acute exercise in mice. Exp Clin Endocrinol Diabetes 115:417-422

25. Fiebig RG, Hollander JM, Ji LL (2001) Exercise down-regulates hepatic fatty acid synthase in streptozotocin-treated rats. J Nutr 131:2252-2259

26. Hoene M, Lehmann R, Hennige AM et al (2009) Acute regulation of metabolic genes and insulin receptor substrates in the liver of mice by one single bout of treadmill exercise. J Physiol 587:241-252

27. Kanehisa M, Araki M, Goto S et al (2008) KEGG for linking genomes to life and the environment. Nucleic Acids Res 36: D480-D484

28. Boppart MD, Aronson D, Gibson L et al (1999) Eccentric exercise markedly increases c-Jun $\mathrm{NH}(2)$-terminal kinase activity in human skeletal muscle. J Appl Physiol 87:1668-1673

29. Patterson KI, Brummer T, O'Brien PM, Daly RJ (2009) Dualspecificity phosphatases: critical regulators with diverse cellular targets. Biochem J 418:475-489

30. Feng J, Tamaskovic R, Yang Z et al (2004) Stabilization of Mdm2 via decreased ubiquitination is mediated by protein kinase B/Aktdependent phosphorylation. J Biol Chem 279:35510-35517

31. Malmlof M, Roudier E, Hogberg J, Stenius U (2007) MEK-ERKmediated phosphorylation of Mdm2 at Ser-166 in hepatocytes. $\mathrm{Mdm} 2$ is activated in response to inhibited Akt signalling. J Biol Chem 282:2288-2296

32. Koyama K, Kaya M, Ishigaki T et al (1999) Role of xanthine oxidase in delayed lipid peroxidation in rat liver induced by acute exhausting exercise. Eur J Appl Physiol Occup Physiol 80:28-33

33. Liu J, Yeo HC, Overvik-Douki E et al (2000) Chronically and acutely exercised rats: biomarkers of oxidative stress and endogenous antioxidants. J Appl Physiol 89:21-28

34. Yung Y, Yao Z, Hanoch T, Seger R (2000) ERK1b, a 46-kDa ERK isoform that is differentially regulated by MEK. J Biol Chem 275:15799-15808

35. Anthony TG, Anthony JC, Lewitt MS, Donovan SM, Layman DK (2001) Time course changes in IGFBP-1 after treadmill exercise and postexercise food intake in rats. Am J Physiol Endocrinol Metab 280:E650-E656

36. Lavoie JM, Fillion Y, Couturier K, Corriveau P (2002) Evidence that the decrease in liver glycogen is associated with the exerciseinduced increase in IGFBP-1. J Appl Physiol 93:798-804

37. Jiang Y, Cypess AM, Muse ED et al (2001) Glucagon receptor activates extracellular signal-regulated protein kinase $1 / 2$ via cAMP-dependent protein kinase. Proc Natl Acad Sci U S A 98:10102-10107

38. Jiang G, Zhang BB (2003) Glucagon and regulation of glucose metabolism. Am J Physiol Endocrinol Metab 284:E671-E678

39. Christensen NJ, Galbo H (1983) Sympathetic nervous activity during exercise. Annu Rev Physiol 45:139-153

40. Coker RH, Krishna MG, Lacy DB, Bracy DP, Wasserman DH (1997) Role of hepatic alpha- and beta-adrenergic receptor 
stimulation on hepatic glucose production during heavy exercise. Am J Physiol 273:E831-E838

41. Kjaer M, Engfred K, Fernandes A, Secher NH, Galbo H (1993) Regulation of hepatic glucose production during exercise in humans: role of sympathoadrenergic activity. Am J Physiol 265: E275-E283

42. Coker RH, Simonsen L, Bulow J, Wasserman DH, Kjaer M (2001) Stimulation of splanchnic glucose production during exercise in humans contains a glucagon-independent component. Am J Physiol Endocrinol Metab 280:E918-E927

43. Coker RH, Koyama Y, Denny JC, Camacho RC, Lacy DB, Wasserman DH (2002) Prevention of overt hypoglycemia during exercise: stimulation of endogenous glucose production independent of hepatic catecholamine action and changes in pancreatic hormone concentration. Diabetes 51:1310-1318

44. Gonzalez B, Manso R (2004) Induction, modification and accumulation of HSP70s in the rat liver after acute exercise: early and late responses. J Physiol 556:369-385

45. Gomez-Cabrera MC, Domenech E, Romagnoli M et al (2008) Oral administration of vitamin $\mathrm{C}$ decreases muscle mitochondrial biogenesis and hampers training-induced adaptations in endurance performance. Am J Clin Nutr 87:142-149

46. Ristow M, Zarse K, Oberbach A et al (2009) Antioxidants prevent health-promoting effects of physical exercise in humans. Proc Natl Acad Sci U S A 106:8665-8670

47. Pratico D, Tangirala RK, Rader DJ, Rokach J, FitzGerald GA (1998) Vitamin E suppresses isoprostane generation in vivo and reduces atherosclerosis in ApoE-deficient mice. Nat Med 4:1189 1192

48. Raney MA, Turcotte LP (2006) Regulation of contraction-induced FA uptake and oxidation by AMPK and ERK1/2 is intensity dependent in rodent muscle. Am J Physiol Endocrinol Metab 291: E1220-E1227

49. Shojaee-Moradie F, Baynes KC, Pentecost C et al (2007) Exercise training reduces fatty acid availability and improves the insulin sensitivity of glucose metabolism. Diabetologia 50:404-413

50. Thamer C, Machann J, Stefan N et al (2008) Variations in PPARD determine the change in body composition during lifestyle intervention: a whole-body magnetic resonance study. J Clin Endocrinol Metab 93:1497-1500 